\title{
Viewpoint: the road to room-temperature conventional superconductivity
}

\author{
Lilia Boeri ${ }^{1, *}$ and Giovanni B. Bachelet ${ }^{1, \dagger}$ \\ ${ }^{1}$ Dipartimento di Fisica, Sapienza Università di Roma, 00185 Roma, Italy
}

(Dated: February 22, 2019)

\begin{abstract}
It is a honor to write a contribution on this memorial for Sandro Massidda. For both of us, at different stages of our life, Sandro was first and foremost a friend. We both admired his humble, playful and profound approach to life and physics. In this contribution we describe the route which permitted to meet a long-standing challenge in solid state physics, i.e. room temperature superconductivity. In less than 20 years the $T_{\mathrm{c}}$ of conventional superconductors, which in the last century had been widely believed to be limited to $25 \mathrm{~K}$, was raised from $40 \mathrm{~K}$ in $\mathrm{MgB}_{2}$ to $265 \mathrm{~K}$ in $\mathrm{LaH}_{10}$. This discovery was enabled by the development and application of computational methods for superconductors, a field in which Sandro Massidda played a major role.

PACS numbers:
\end{abstract}

Since K.H. Onnes discovered in 1911 that a sample of mercury, cooled below a critical temperature $\left(T_{\mathrm{c}}\right)$ of $4 \mathrm{~K}$, exhibits a vanishing resistivity, it became immediately clear that such a superconductivity, realized at ambient conditions, could have spectacular electrical network applications. A related unique property of superconductors, perfect diamagnetism, is equally attractive, because quantum levitation paves the road to futuristic scenarios, such as levitating vehicles.

However, more than 100 years after the original discovery, none of these large-scale applications has advanced to a point where it is economically viable. Currently, the only applications of superconductors are found in devices and facilities whose cost is not an issue: superconducting magnets are used in large-scale particle accelerators and storage rings, diagnostical devices, antennas etc. The most severe obstacle to cost-sensitive applications are the prohibitive refrigeration costs required to cool the existing materials below their critical temperatures. The only compounds which superconduct above the liquid nitrogen $\left(\mathrm{N}_{2}\right)$ boiling point, the high- $T_{\mathrm{c}}$ cuprates, have several characteristics which make them not suitable for large scale applications: being brittle, they have high manufacturing costs; the presence of magnetism in the phase diagram and the exotic symmetry of the superconducting gap make them sensitive to grain boundaries. [1]

As a result, within today's technological applications, the most used superconductors are simple intermetallic alloys, with $T_{\mathrm{c}}$ 's in the range of $10-30 \mathrm{~K}$. In addition to being much cheaper and easier to manipulate, these superconductors are also easier to describe theoretically: unlike the exotic cuprates, they are described to a high degree of accuracy by the theory of conventional phononmediated superconductivity, developed in the 60's; the progress of current ab-initio methods to calculate the electron-phonon coupling in the last 20 years is such that the normal- and superconducting-state properties of actual materials can now be computed to a high degree of accuracy based on the sole knowledge of their chemical composition and crystal structure; actually, once the formula unit is given, the equilibrium crystal structure itself may be predicted, to a great degree of accuracy, using a combination of ab-initio structural energies and modern optimization methods, a valuable theoreticalcomputational tool which becomes almost indispensable when one or some of the chemical components are light elements which are hard to locate by X-ray diffraction.

The availability of quantitative methods to describe the electronic, vibrational, and structural properties of materials has granted not only an accurate understanding of many existing superconductors, but also the exploration and prediction of hypothetical, new ones, up to providing, last year, the solution to a puzzle previously deemed impossible: a room-temperature superconductor. In the summer of 2018, two different groups reported the discovery of a $T_{\mathrm{c}}$ of $260 \mathrm{~K}$ in a sample of lanthanum hydride $\left(\mathrm{LaH}_{10}\right)$, pressurized to Megabar pressures $(\sim 150$ GPa) [2-4], and already three years before another highpressure hydride, $\mathrm{SH}_{3}$, had set the all-time superconducting record with a $T_{\mathrm{c}}$ of $203 \mathrm{~K} \mathrm{[5];} \mathrm{in} \mathrm{both} \mathrm{cases} \mathrm{their}$ stability and superconductive nature, and to a very good approximation their $T_{\mathrm{c}}$, had been predicted by ab-initio calculations. [6, 7]

The discovery of high- $T_{\mathrm{c}}$ superconductivity in highpressure hydrides does not solve any technological problem, since, in daily life, to achieve pressures million times larger than ambient pressure is much more difficult than refrigerating samples down to a few K. Nonetheless, the discovery of room-temperature superconductivity in $\mathrm{LaH}_{10}$ has smashed several psychological barriers, showing: $(i)$ that superconductivity may be achieved at ambient temperature; $(i i)$ that high- $T_{\mathrm{c}}$ superconductivity may be achieved by the conventional (electron-phonon) mechanism; (iii) that, using first-principles calculations, new superconductors may be reliably predicted (and designed) before experiments.

Obviously, the achievement of room-temperature superconductivity was not a matter of sheer luck, but rather the result of a long process, which experienced a strong acceleration at the beginning of this century. The aim 


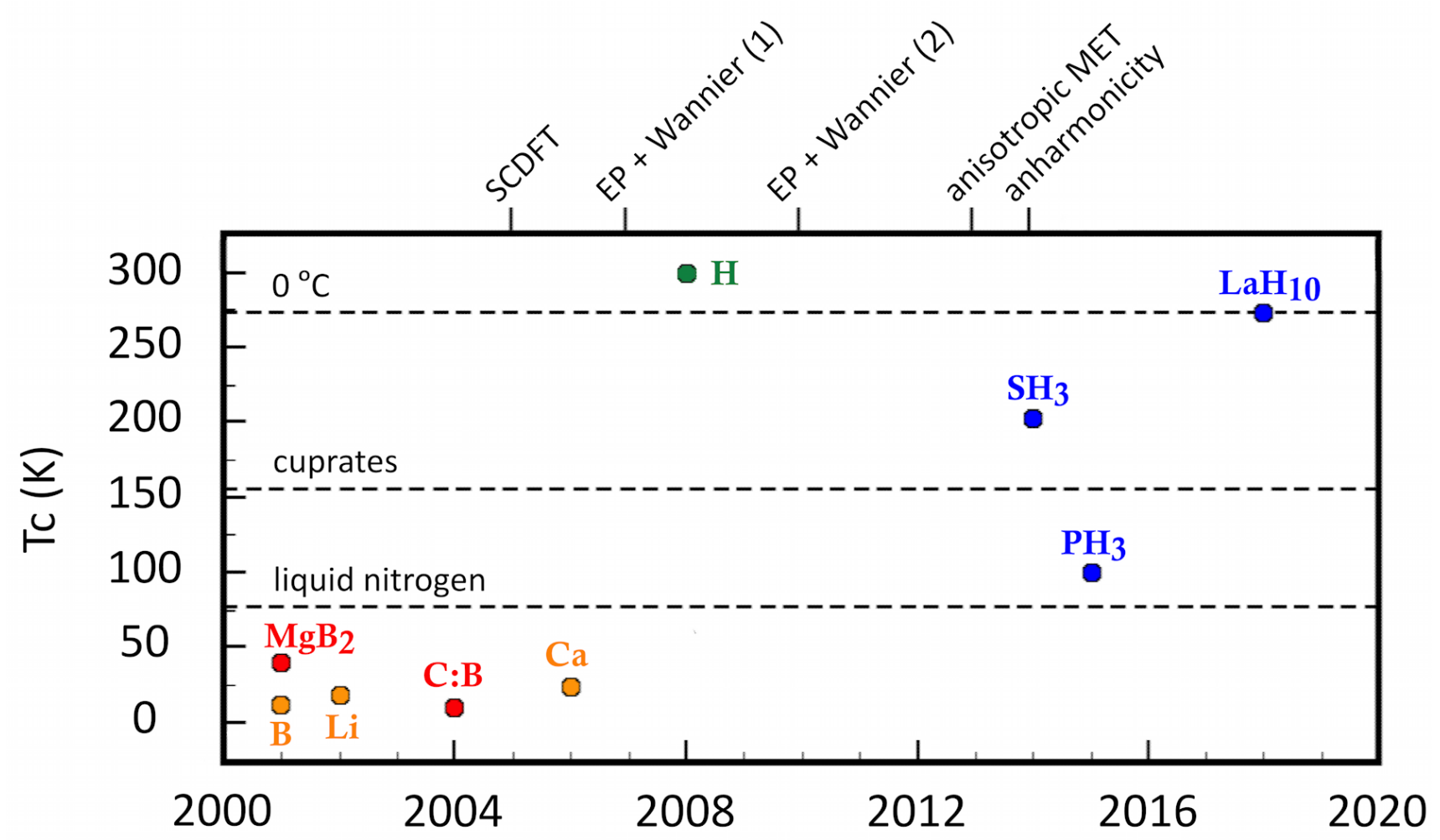

Figure 1. The main four milestones on the route to room-temperature superconductivity in the 21 st century: discovery of $\mathrm{MgB}_{2}$ and other covalent superconductors (red); elemental superconductors at high pressures (orange); theoretical prediction of the phase diagram and superconductivity in metallic hydrogen (green); superconductivity in metallic hydrides at high pressure (blue). The tics on the top axis mark crucial developments in the field of computational superconductivity, a field pioneered by Sandro Massidda and collaborators: Superconducting Density Functional Theory (SCDFT) [8, 9]; e-ph interaction with Wannier functions [10, 11]; anisotropic Migdal-Eliashberg Theory [12]; self-consistent calculation of anharmonic effects on phonon spectra [13].

of this viewpoint is to illustrate the last steps of this process, which took place in the last 20 years. Out of the rich literature on the subject, we have identified four milestones, illustrated in Fig. 1, which marked the route to the $\mathrm{LaH}_{10}$ discovery.

1. The discovery of $\mathrm{MgB}_{2}$ and other covalent superconductors (2001-), red circles.

2. The study of elemental superconductors at high pressures (2002-), orange circles.

3. The theoretical calculation of the phase diagram and $T_{\mathrm{c}}$ 's of metallic Hydrogen. (2007-), green circle.

4. The prediction of superconductivity in highpressure hydrides (2008-), blue circles.

The two last milestones are directly related to two brilliant intuitions on solid hydrogen and hydrogen-rich hydrides due to Neil Ashcroft [14, 15] which we will discuss later. It is also interesting to note that, at the start of the route, the experimental discovery of the remarkable superconducting $T_{\mathrm{c}}$ of $\mathrm{MgB}_{2}$ preceded its understanding in terms of $a b$ initio electronic bands and phonons, whereas, towards the end of the route, theoretical predictions based on large-scale $a b$ initio calculations led experimental groups to synthesize previously unknown hydrides like $\mathrm{SH}_{3}$ or $\mathrm{LaH}_{10}$, eventually confirming both their stability and their exceptionally high $T_{\mathrm{c}}$. In other words, the development and extensive application of $a b$ initio methods was so impetuous as to turn them, in less than 20 years, from valuable instruments for the interpretation of existing superconductors into reliable forecasting tools for new superconductors. Before entering the route leading to the discovery of $\mathrm{LaH}_{10}$, we need, however, to rapidly recall the basic theoretical background of superconductivity, a field to which Sandro, with his students and collaborators, gave an essential contribution.

The simplest microscopic theory of superconductivity, the Bardeen-Cooper-Schrieffer (BCS) theory, describes the superconducting state as a state of macroscopic quantum coherence, where electrons form pairs of opposite spin and momentum (Cooper Pairs) held together by an attractive glue. The superconducting state is character- 
ized by the presence of a gap $\Delta$ in the superconducting spectrum, which closes at the critical temperature $T_{\mathrm{c}}$; above $T_{\mathrm{c}}$, conventional superconductors behave as normal metals. In the original BCS paper, the glue is provided by lattice vibrations (phonons), but other mediators are in principle possible, such as plasmons, spin fluctuations, charge fluctuations, etc.

The strong-coupling extension of BCS theory, known as Migdal-Eliashberg (ME) theory, is a diagrammatic theory for interacting electrons and bosons [16]. At the heart of the theory is the so-called (isotropic) electronphonon spectral function, defined as:

$\alpha^{2} F(\omega)=\frac{1}{N\left(E_{F}\right)} \sum_{\mathbf{k q}, \nu}\left|g_{\mathbf{k}, \mathbf{k}+\mathbf{q}, \nu}\right|^{2} \delta\left(\epsilon_{\mathbf{k}}\right) \delta\left(\epsilon_{\mathbf{k}+\mathbf{q}}\right) \delta\left(\omega-\omega_{\mathbf{q}, \nu}\right)$,

where $N\left(E_{F}\right)$ is the DOS at the Fermi level, $\omega_{\mathbf{q}, \nu}$ is the phonon frequency of mode $\nu$ and wave vector $\mathbf{q}$, and $\left|g_{\mathbf{k}, \mathbf{k}+\mathbf{q}, \nu}\right|$ is the $e-p h$ matrix element between two electronic states of wave vectors $\mathbf{k}$ and $\mathbf{k}+\mathbf{q}$ at the Fermi level. The expression of the e-ph spectral function $\alpha^{2} F(\omega)$ clearly evidences why superconductors are so hard to predict (or why superconducting properties are so sentitive to small details of the electronic structure of a given material): since in Eq. (1) the phonon spectrum is weigthed by the $e-p h$ matrix elements $\left|g_{\mathbf{k}, \mathbf{k}+\mathbf{q}, \nu}\right|$, which can be very different for different phonon modes, and since the double delta function $\delta\left(\varepsilon_{\mathbf{k}}^{n}\right) \delta\left(\varepsilon_{\mathbf{k}+\mathbf{q}}^{m}\right)$ restricts the sum of $e-p h$ matrix elements to electronic states at the Fermi level, only a small fraction of the full electronic and phononic spectrum contributes to superconductivity.

To understand and predict new conventional superconductors essentially amounts to explaining or conceiving materials where electrons and phonons are strongly coupled (large $\left|g_{\mathbf{k}, \mathbf{k}+\mathbf{q}, \nu}\right|$ ), which can be done with considerable accuracy since all quantities entering the $e$ - $p h$ spectral function can be computed from first-principles within Density Functional Perturbation Theory [17]. Once the $e-p h$ spectral function is known, the critical temperature of a material can be computed to different degrees of sophistication. The simplest approach adopts an approximate formula for $T_{\mathrm{c}}$; a popular choice for phononmediated superconductors is the Mc-Millan-Allen-Dynes formula [18]

$$
T_{c}=\frac{\omega_{\log }}{1.2 k_{B}} \exp \left[-\frac{1.04(1+\lambda)}{\lambda-\mu^{*}(1+0.62 \lambda)}\right], \text { where }
$$

$\lambda=2 \int d \omega \frac{\alpha^{2} F(\omega)}{\omega}$ and $\omega_{\log }=\exp \left[\frac{2}{\lambda} \int \frac{d \omega}{\omega} \alpha^{2} F(\omega) \ln (\omega)\right]$ are the $e$-ph coupling constant and logarithmic-averaged phonon frequency; $\mu^{*}$ is the so-called Morel-Anderson pseudopotential, obtained by screening the full Coulomb potential up to a characteristic cut-off energy. [19]

This approach neglects effects which may influence the behavior of a superconductor, such as possible anisotropies of the superconducting gap, strong-coupling corrections to the $T_{\mathrm{c}}$ expression, self-energy effects on the electronic and phonon spectra, etc. These effects may be handled from first principles in two ways: $(i)$ on one hand, the so-called anisotropic Migdal-Eliashberg Theory (AMET), which solves the anisotropic Migdal-Eliashberg equations, computing the full electronic and phonon selfenergies; although very accurate, it cannot be considered a fully ab-initio theory, but rather an advanced combination of many-body techniques and first-principles ingredients; (ii) on the other hand, the superconducting Density Functional Theory (SCDFT) proposed by Oliveira, Gross and Kohn in 1988 [20] but implemented for real materials only at the beginning of the 2000's by Sandro Massidda and Hardy Gross' groups, a conceptually different extension of the DFT approach to the the superconducting state $[8,9]$.

Both AMET and SCDFT remove two strong assumptions of the simpler ME version, i.e. the isotropy of the $e-p h$ coupling and superconducting gap and the use of an empirical Morel-Anderson pseudopotential. Excellent reviews of the latest developments of the two approaches may be found in Refs. 12-21, and 22, respectively. For our historical perspective, it is sufficient to say that the two approaches have by now reached comparable accuracy ( $\sim 10 \%$ on the $T_{\mathrm{c}}$ ), and that a formal mapping of the two theories is possible using the Sham-Schlüter connection [23].

The first attempt to calculate the $T_{\mathrm{c}}$ of a conventional superconductor from first principles dates back to the 1970's, when Gaspary and Gyorffy proposed an approximate method to compute the $e-p h$ coupling in superconductors [24]. The deformation-potential approach by Kahn and Allen is ten years older [25]. Both approaches were too crude to yield reliable results; a serious limitation was represented by the prohibitive cost of computing phonon spectra in those years: they were fitted to experiments via semi-empirical force-constant models.

A big progress came with the development of Density Functional Perturbation Theory (DFPT) [17]. On this basis, Savrasov and Savrasov published in 1996 the first complete set of $T_{\mathrm{c}}$ calculations, showing that DFPT, combined with a semi-phenomenological ME approach, was capable of a reliable description of the superconducting and transport properties for all known elemental superconductors at ambient pressure, and also of the explanation why some elements, like $\mathrm{Cu}$ or $\mathrm{Pd}$, are not superconductors [26]. The importance of this work was recognized only a few years later, because, at that time, the superconductivity debate was dominated by the cuprates, where such a conventional e-ph mechanism for the high$T_{\mathrm{c}}$ had been clearly ruled out [27].

Moreover, an old semiempirical argument based on the simplest version of the ME theory predicted an upper limit of $25 \mathrm{~K}$ for the $T_{\mathrm{c}}$ of conventional superconductors -the so-called Cohen-Anderson limit- and this seemed to be confirmed by the experimental discoveries: year by 
year $T_{\mathrm{c}}$ had been slowly and painfully pushed up to $25 \mathrm{~K}$ in the best superconductors known at that time, the A15, $\left(\mathrm{Nb}_{3} \mathrm{Ge}\right)$, and it seemed impossible to go beyond that value; cuprates were not an exception to this rule, since their superconductivity was soon recognized as due to a coupling mechanism other than e-ph coupling.

This is why, in our recollection of the path to roomtemperature superconductivity, a much less spectacular discovery paradoxically appears more important than that of cuprates: in 2001, a simple intermetallic, magnesium diboride $\left(\mathrm{MgB}_{2}\right)$, was found to be superconducting with a $T_{\mathrm{c}}$ of $39 \mathrm{~K}$. [28] Besides disproving the CohenAnderson limit for $T_{\mathrm{c}}, \mathrm{MgB}_{2}$ has characteristics which make it stand out of the group of previously known conventional superconductors: its superconducting gap has very different values on different sheets of its Fermi surface (two-gap superconductivity) and its phonon spectrum is strongly anharmonic.[29] Fortunately the description of such effects requires a relatively straightforward extension of the standard theory of conventional superconductivity which can be incorporated into ab-initio approaches: [30-33] the $\mathrm{MgB}_{2}$ case induced important methodological developments, such as the implementation of the anisotropic formalism for $e$-ph interaction, the development of Wannier-function methods to accurately compute linewidths and Kohn anomalies, a new formalism to compute self-consistently anharmonic effects on phonon spectra, etc. $[10,11,13]$ But the most important role played by the $\mathrm{MgB}_{2}$ discovery was probably to provide a new playground for the understanding of material-specific mechanisms which within the conventional $e$-ph coupling may give rise to a high $T_{\mathrm{c}}$.

Until 2001, the best conventional superconductors were intermetallics containing $d$ metals, and the main strategy adopted to "boost" their $T_{\mathrm{c}}$ was the use of different dopants to increase their density of states at the Fermi level. Unlike them, $\mathrm{MgB}_{2}$ was a simple $s-p$ material whose high- $T_{\mathrm{c}}$ derived from "covalent bonds driven metallic", as nicely synthesized by the title of a paper by Warren Pickett [34].

The meaning of these expressions is most easily understood if one rewrites the $e-p h$ coupling constant $\lambda$ using the simplified Hopfield expression:

$$
\lambda=\frac{N(0) I^{2}}{M \omega^{2}}, \text { where }
$$

$N(0)$ is the density of states at the Fermi level, $I$ the e-ph matrix element, and $M \omega^{2}$ a phonon force constant.

In most elemental superconductors and, to a lesser extent, in A15 compounds, the $e-p h$ coupling spreads over several phonon modes and electronic states; the highest $T_{\mathrm{c}}$ 's are obtained from the largest $N(0)$ values (typically within narrow transition-metal bands) combined with a moderate average $e-p h$ matrix element and relatively low phonon frequencies, and remain limited to $\sim 25 \mathrm{~K}$.
On the other hand, $\mathrm{MgB}_{2}$ (and also, as we shall later see, some high-pressure hydrides) have relatively small $N(0)$ 's. What drives the high $T_{\mathrm{c}}$, in these cases, are large e-ph matrix elements $(I)$ between a few selected bond-stretching phonons and the electrons which contribute to those directional, covalent bonds. However, such electrons are shared by neighboring atoms within fully occupied bonding states which are well separated in energy from the corresponding, empty antibonding states; as a result, under normal external pressure, almost all covalent or covalent-polar solids are insulators with $N(0)=0$. The few known exceptions to the thumb rule "covalent materials are insulators" all turned out to be superconductors, with higher or lower $T_{\mathrm{c}}$ 's primarily depending on how large or small is $N(0): \mathrm{MgB}_{2}(\sim 40 \mathrm{~K})$, B-doped insulators $(\sim 10 \mathrm{~K})[35,36]$, and also hypothetical compounds like hole-doped LiBC or graphane [37, 38] for which $T_{\mathrm{c}}$ 's of $\sim 100 \mathrm{~K}$ were theoretically predicted. Briefly, the experimental discovery of $\mathrm{MgB}_{2}$ induced a fresh-mind theoretical re-examination of the balance among different material-specific ingredients of a high$T_{\mathrm{c}}$ conventional superconductor, which in turn ignited a big hunt for covalent and light-element superconducting compounds. In a few years, several classes of new (conventional) superconductors were experimentally discovered and theoretically interpreted with ab-initio calculations [39-41].

The above discoveries are very important, but not directly related to the recent achievement of roomtemperature superconductivity, so here we will not discuss them any further. We will, instead, shift to a topic which is at first sight unrelated to superconductivity.

In the same years as the $\mathrm{MgB}_{2}$ discovery, major progresses took place in the field of high pressure research [42]. Better diamond anvil cells (DAC) equipped with innovative experimental setups allowed to perform in-situ resistivity, susceptibility, Raman and IR-spectra measurements up to pressures in the Megabar range. As a result, in the first years of the $21^{\text {st }}$ century the phase diagram of many compounds was explored to an unprecedented degree of accuracy. As more and more information on the behavior of matter under extreme pressure conditions piled up, it became clearer and clearer that, under pressure, even the structural evolution of the simplest elemental solids defies the chemical understanding based on ambient-pressure experience. Widely accepted concepts, such as the idea that, under sufficiently high pressure, all elements eventually form close-packed structures, urgently needed to be revised.

A 2005 survey of the superconducting properties of all elemental solids shows that, under appropriate conditions, almost all elements of the Periodic Table can be made superconducting, including those which are insulating at ambient pressure, like silicon, boron or oxygen [43].

Sandro's group has played an important role in highpressure superconducting research since its early days, 
when it appeared a mere intellectual curiosity. Highpressure experiments looked like an ideal testbed for the newly-developed SCDFT method. Particularly instructive, in this respect, are his early works on lithium and other alkali metals under pressure, which discuss in detail the role of phonon softening and demonstrate an anomalously strong role of the residual electronic screening in suppressing the $T_{\mathrm{c}}$ [44]. Sandro's interest in high pressure remained high; his most recent work on superconductivity in sulfur appeared in 2017 [45].

A development which was crucial to interpret highpressure superconductivity experiments was ab-initio crystal structure prediction (CSP), which was also developed in the same years [46]. The basic idea of CSP is to use efficient search and optimization methods to find the global minimum of a complex energy landscape, representing a given compound at given external conditions. If the search space is sufficiently large, these methods often permit to identify the actual ground-state structure of a system. Such a possibility to predict the crystal structure of a material from first principles is particularly attractive when diffraction experiments are difficult or impossible to perform. Two of the early successes of $a b$-initio crystal structure prediction were the identification of the high-pressure superconducting phases of boron $\left(\max T_{\mathrm{c}} 11 \mathrm{~K}\right)$ [47] and calcium $\left(T_{\mathrm{c}} 25 \mathrm{~K}\right)$ [48], two light elements with low X-Ray cross sections. After their equilibrium crystal structures were determined by CSP methods, $a b$ initio calculations reproduced their experimental $T_{\mathrm{c}}$ 's with an accuracy of a few $\mathrm{K}$, as well as their experimental pressure behavior $[49,50]$.

In the various "Periodic Tables of Superconducting Elements" published over the last ten years the first element, hydrogen, is always missing. The reason is practical: the pressure required to metallize hydrogen is at the limit of todays' capabilities.

But as soon as the (huge) metallization pressure is reached, metallic hydrogen is expected to be a superconductor, and a good one, with very high $T_{\mathrm{c}}$. The original argument, proposed by Neil Ashcroft as early as 1968 [14], can be understood on the basis of Eq. 2: (i) Due to its low atomic mass, the characteristic frequencies of hydrogen are high (prefactor $\omega_{\log }$ ); (ii) Due to the lack of screening from core electrons, electron-ion matrix elements are high (exponent $\lambda$ ); this means that, even with a moderate $N(0), T_{\mathrm{c}}$ 's can be quite high. Ashcroft's argument is so general, that it does not require any specific assumption on the crystal structure of hydrogen. Based on the simplified understanding of elemental crystals under pressure, early ab-initio calculations of the superconductivity of metallic hydrogen typically assumed fcc structures, and predicted $T_{\mathrm{c}}$ 's as high as $600 \mathrm{~K}[51,52]$.

The actual high-pressure phase diagram of hydrogen turned out to be, however, much more complex than initially thought. A very important step in its understanding is a computational study of the Cambridge group, published in 2007 and based on ab-initio random structure searches, identified several high-pressure phases for which only limited spectroscopic information was, at the time, available [53].

At ambient pressure, hydrogen forms a molecular crystal in which the $\mathrm{H}_{2}$ molecules are loosely arranged on a disordered lattice; in this structure, hydrogen exhibits a gap as large as $10 \mathrm{eV}$. Under increasing pressure, the $\mathrm{H}_{2}$ molecules tend to arrange on more and more regular lattices, giving rise to a sequence of phase transitions. In one of these molecular structures, a band-overlap insulatorto-metal transition is predicted to occur at $\sim 400 \mathrm{Gpa}$. Slightly after (at $\sim 500 \mathrm{GPa}$ ), another transition is predicted to occur towards an atomic $(\beta-S n)$ structure in which the molecular units are dismantled; this structure is also metallic.

In 2008, Sandro's group published what can be considered the first ab-initio calculation of the $T_{\mathrm{c}}$ of metallic hydrogen in a physically-meaningful structure [54-56]. The calculation assumes a Cmca structure, a simplified version of the intermediate high-pressure molecular structures in which one can still identify two different inter and intra-molecular bonds. As the length of these two types of bonds becomes comparable, the gap between the bonding and antibonding states closes, and hydrogen undergoes an insulator-to-metal transition. The resulting Fermi surface comprises both holes (bonding) and electron (antibonding) pockets. According to Sandro and collaborators, this should lead to a complex three-band structure of the superconducting gap. The corresponding predicted $T_{\mathrm{c}}$ 's (up to $300 \mathrm{~K}$ at $500 \mathrm{GPa}$ ) were one order of magnitude higher than what had been observed in other elements, confirming on a quantitative basis Ashcroft's 1968 intuition that, at least in principle, high- $T_{\mathrm{c}}$ conventional superconductivity was not impossible. More recent calculations for the atomic $\beta-S n$ phase predict an equally large $T_{\mathrm{c}}$ 's. $[57,58]$

Until a few years ago the metallization of hydrogen was beyond reach, but at least three experimental groups have reported it in the last two years, at pressures ranging from 360 to $500 \mathrm{GPa}$ (3.6 to 5 Mbar)[59-61]; the crystal structure of the metallic phase and hence the mechanism of metallization remain, however, controversial. Direct evidence is missing and computed transition pressures have a large uncertainity due to quantum lattice effects, which in hydrogen may strongly affect the relative stability of different phases as well as the phonon spectra [62,63]. Aside from its experimental realization, which looks closer and closer, the main relevance of superconducting hydrogen is not practical, because of the huge metallization pressure required, but rather theoretical, as the starting idea whose developments eventually led to the first actual discovery of conventional high- $T_{\mathrm{c}}$ superconductivity.

At the beginning of the century covalent hydrides were intensely studied for their ability to incorporate and re- 
lease hydrogen under appropriate conditions of temperature and pressure [64]. Efficient hydrogen-storage materials are at the heart of hydrogen fuel cells, a clean alternative to fossil fuels for on-board automotive applications. They received a strong attention in a period when, for political and technological reasons, it seemed highly likely that the growing demand for fossil fuels would be hard to meet in the near future - See Ref. 65.

In 2004, Ashcroft conjectured that the ability of these hydrides to trap a large fraction of hydrogen in a host lattice could be exploited to exert a chemical pressure on hydrogen, thus lowering its metallization pressure [15]. The first experiments on silane $\left(\mathrm{SiH}_{4}\right)$ were perfomed in 2008 and confirmed Ashcroft's intuition, but $T_{\mathrm{c}}$ 's were disappointingly low [66]. Such a low $T_{\mathrm{c}}$ did not imply that the strategy to metallize hydrogen using hydrides was wrong per se. In hydrides, just like in other classes of superconductors, small details of the electronic structure which depend on crystal structure, chemical composition, doping, and other intrinsic or external conditions, would probably have a crucial impact on $T_{\mathrm{c}}$. Superconductivity was indeed close, but the chemical composition yielding a high- $T_{\mathrm{c}}$ hydride was not as trivial as originally hoped.

At this point, the experience gained with elemental solids at high pressure turned out to be extremely precious: it had been convincingly demonstrated that, in fact, ab-initio methods for crystal structure prediction, combined with methods to compute the critical temperatures of superconductors, could be used to calculate accurate phase diagrams. Hydrides, being binary systems, pose the additional complication that several compositions may form and cohexist as a function of pressure; however, estimating the relative stability of different compositions by first-principles calculations through the convex hull constructution is rather straightforward [67].

In the years immediately following Ashcroft's prediction, many binary hydrides were computationally investigated, in hope to identify prospective high- $T_{\mathrm{c}}$ superconductors. A very important milestone in this process is a paper by Zurek et al., published in 2009, where, for the first time, it was explicitly pointed out that offstoichiometry phases of hydrides, in particular the superhydrides which form at high pressure, exhibit very different properties from their ambient-pressure counterparts, and some of them also show high- $T_{\mathrm{c}}$ superconductivity [68].

The real breakthrough came in 2015, when, for the first time, a $T_{\mathrm{c}}$ exceeding $200 \mathrm{~K}$ was measured in pressurized $\mathrm{SH}_{3}$, which forms when sulfur di-hydride $\left(\mathrm{SH}_{2}\right)$ is placed in a hydrogen-rich atmosphere and compressed in a DAC above $\sim 10 \mathrm{GPa}$ [69]. The $\mathrm{H}_{2} \mathrm{~S}$ molecule, which is analogous to water, at ambient pressure forms a molecular, insulating crystal; in the low-pressure phases of $\mathrm{SH}_{3}$, also insulating, hydrogen is trapped in molecular form $\left(\mathrm{H}_{2}\right)$ in the open $\mathrm{H}_{2} \mathrm{~S}$ lattice. As pressure is increased above $20 \mathrm{GPa}$, hydrogen is gradually incorporated into the $\mathrm{SH}_{2}$ lattice, and, at $\sim 100 \mathrm{GPa}, \mathrm{SH}_{3}$ units start to form. This causes a gradual insulator-to-metal transition. At 150 GPa the S-H interaction becomes so strong that $\mathrm{SH}_{3}$ orders in a highly-symmetric $(b c c)$ structure with a rather unusual bonding: sulfur forms covalent bonds with its three hydrogen neighbours. In analogy to $\mathrm{MgB}_{2}$, these bonds, driven metallic, are at the origin of the high- $T_{\mathrm{c}}$ conventional superconductivity of $\mathrm{SH}_{3}[70,71]$.

The discovery of high- $T_{\mathrm{c}}$ superconductivity in $\mathrm{SH}_{3}$ is an impressive demonstration of the progress of current high-pressure experimental techniques, but it also represents the first unquestionable breakthorugh of the $a b$ initio approach to the search and discovery of new superconductors: it was, in fact, a theoretical DFT paper [72] which, a few months before the experimental report, predicted the exact pressure, structure, $T_{\mathrm{c}}$ and chemical composition of superconducting $\mathrm{SH}_{3}$.

This was not the only succesful prediction of high$T_{\mathrm{c}}$ superconductivity in high-pressure hydrides. A few months later, several theoretical papers pointed out that the neighbour of sulfur in the Periodic Table, phosphorus, should also form superconducting hydrides at high pressures, with $T_{\mathrm{c}}$ 's of $\sim 100 \mathrm{~K}$, but also that all these superconducting phases would be metastable with respect to elemental decomposition [73-75]. The metastability of such samples was later confirmed experimentally [74, 76].

Even more impressive is the case of lanthanum: in 2017 a computational study predicted that it forms superconducting superhydrides with $T_{\mathrm{c}}$ 's as large as $300 \mathrm{~K}$ [7], and in the summer of 2018 two different groups reported that one of these superhydrides, $\mathrm{LaH}_{10}$, does indeed form in a DAC, and their highest reported $T_{\mathrm{c}}(265 \mathrm{~K})$ is close to room temperature.

$\mathrm{LaH}_{10}$ belongs to a larger class of sodalite-like clathrates of chemical formula $\mathrm{XH}_{n}$ which, according to theoretical predictions, should also include the hydrides of yttrium, scandium, magnesium, calcium and several rare earths $[7,77]$. In these compounds hydrogen forms interconnected polyhedral cages each enclosing a guest atom $X$, yielding a dense sponge-like hydrogen lattice in which the shortest $\mathrm{H}-\mathrm{H}$ distances are close to those predicted for metallic atomic hydrogen in the $\beta$-Sn structure. In fcc $\mathrm{LaH}_{10}$, for example, each lanthanum sits in the middle of a 32-hydrogen cage; in bcc $\mathrm{LaH}_{6}$, the lanthanum's nearest neighbors are 24 hydrogen atoms; and so on. Many such superhydrides with a sodalite-like clathrate structure have been predicted to exhibit high- $T_{\mathrm{c}}$ conventional superconductivity, the highest $T_{\mathrm{c}}$ 's being expected for yttrium, calcium and magnesium [78].

After the spectacular demonstration of their predictive power not only for the new high- $T_{\mathrm{c}}$ compound $\mathrm{SH}_{3}$, but also for the possibility of room-temperature conventional superconductivity in $\mathrm{LaH}_{10}$, a natural question to ask is: what is the next goal of electronic-structure methods?

In our view, the first aim is to understand why some hydrides achieve room-temperature superconductivity, 
while others don't. This is a step beyond the state-ofthe-art ability of computing and successfully predicting phase diagrams and superconductive $T_{\mathrm{c}}$ 's of different hydrides from first principles, and amounts to identifying the specific mechanisms leading to high- $T_{\mathrm{c}}$ in different hydrogen compounds.

First of all, one has to acknowledge the evidence of qualitatively different families within the high-pressure hydrides. For example, the high- $T_{\mathrm{c}}$ superconductivity mechanism cannot be the same in $\mathrm{SH}_{3}$ and $\mathrm{LaH}_{10}$ : at variance with $\mathrm{SH}_{3}$, where sulfur is incorporated into the $\mathrm{H}$ sublattice, forming covalent $\mathrm{S}-\mathrm{H}$ bonds, the main role of the guest atom in sodalite-like clathrates is to provide charge and stabilize the the $\mathrm{H}$ sublattice by "filling the holes" in the sponge-like geometry.

Then one has to recognize that $\mathrm{H}-\mathrm{H}$ distances close to those realized in atomic $\beta$-Sn hydrogen are a necessary, but not sufficient condition for high- $T_{\mathrm{c}}$ superconductivity. For example, when first synthesized, the crystal structure of $\mathrm{FeH}_{5}$ (which can be seen as a cubic $\mathrm{FeH}_{3}$ lattice intercalated by two-dimensional $\mathrm{H}$ layers), seemed a promising candidate for high- $T_{\mathrm{c}}$ superconductivity [79], because of the propitious $\mathrm{H}-\mathrm{H}$ distances in the intermediate layer; first-principles calculations demonstrated that, instead, the Fe-H bonds which dominate the electronic states at the Fermi level are too weak to support it [80].

The second aim, directly connected to the first, is to understand whether and how superconductivity in highpressure hydrides can have any impact on actual applications. This obviously means to seek and achieve a substantial reduction of the metallization and superconducting pressure, a goal which may be sought according to different strategies:

- already known hydrides may be used a starting point for optimization strategies. For example, in sodalite-like clathrates, the evident correlation between the size and valence of the guest atom and the stabilization pressure may be systematically studied and exploited to lower the effective metallization pressure [81];

- ternary hydrides may offer an even larger flexibility to stabilize different crystalline superhydride phases [82], or to achieve metallization at lower pressure via doping of molecular phases [83, 84];

- compounds containing light elements other than hydrogen, although their high-pressure behavior can be quite different from hydrides, [85] may also be made superconducting under high pressure [86]; among them the elements forming covalent bonds at ambient pressure, like boron and carbon, are particularly promising, since, when doped, these bonds have already proved to support high $T_{\mathrm{c}}$. As a matter of fact, the current record for conventional superconductivity at ambient pressure $(56 \mathrm{~K})$ has been achieved last year in amorphous boron-doped carbon [87], and even higher $T_{\mathrm{c}}$ 's have been predicted in (ambient-pressure) doped hydrogenated graphene (graphane) [38].

In summary, first-principles calculations of the superconducting properties of conventional superconductors, a field of research pioneered by Sandro Massidda, have by now gained the status of a reliable and efficient tool not only to interpret existing superconductors, but also to predict new ones, and are likely to play a decisive role in the strategies aimed at achieving ambient-pressure high$T_{\mathrm{c}}$ superconductors.

The authors acknowledge support from Fondo AteneoSapienza 2017.

*lilia.boeri@uniroma1.it

$\dagger$ giovanni.bachelet@uniroma1.it

[1] A. Gurevich, Nature Materials 10, 255 EP (2011).

[2] A. P. Drozdov, V. Minkov, S. Besedin, P. Kong, M. Kuzovnikov, D. Knyazev, and M. Eremets, arXiv preprint, arXiv:1808.07039 (2018).

[3] M. Somayazulu, M. Ahart, A. K. Mishra, Z. M. Geballe, M. Baldini, Y. Meng, V. V. Struzhkin, and R. J. Hemley, Phys. Rev. Lett. 122, 027001 (2019).

[4] A. Drozdov and et al., arXiv preprint, arXiv:1812.01561 (2018).

[5] A. P. Drozdov, M. I. Eremets, I. A. Troyan, V. Ksenofontov, and S. I. Shylin, Nature 525, 73 (2015).

[6] D. Duan, Y. Liu, F. Tian, D. Li, X. Huang, Z. Zhao, H. Yu, B. Liu, W. Tian, and T. Cui, Sci. Rep. 4 (2014).

[7] F. Peng, Y. Sun, C. J. Pickard, R. J. Needs, Q. Wu, and Y. Ma, Phys. Rev. Lett. 119, 107001 (2017).

[8] M. Lüders, M. A. L. Marques, N. N. Lathiotakis, A. Floris, G. Profeta, L. Fast, A. Continenza, S. Massidda, and E. K. U. Gross, Phys. Rev. B 72, 024545 (2005).

[9] M. A. L. Marques, M. Lüders, N. N. Lathiotakis, G. Profeta, A. Floris, L. Fast, A. Continenza, E. K. U. Gross, and S. Massidda, Phys. Rev. B 72, 024546 (2005).

[10] F. Giustino, M. L. Cohen, and S. G. Louie, Phys. Rev. B 76, 165108 (2007).

[11] M. Calandra, G. Profeta, and F. Mauri, Phys. Rev. B 82, 165111 (2010).

[12] F. Giustino, Rev. Mod. Phys. 89, 015003 (2017).

[13] I. Errea, M. Calandra, and F. Mauri, Phys. Rev. B 89, 064302 (2014).

[14] N. Ashcroft, Phys. Rev. Lett. 21, 1748 (1968).

[15] N. Ashcroft, Phys. Rev. Lett. 92, 187002 (2004).

[16] P. B. Allen and B. Mitrović, Theory of Superconducting Tc, Solid State Physics, Vol. 37 (Academic Press, 1983) pp. $1-92$.

[17] S. Baroni, S. de Gironcoli, A. Dal Corso, and P. Giannozzi, Rev. Mod. Phys. 73, 515 (2001).

[18] P. Allen and R. Dynes, Phys. Rev. B 12, 905 (1975).

[19] P. Morel and P. W. Anderson, Phys. Rev. 125, 1263 (1962).

[20] L. N. Oliveira, E. K. U. Gross, and W. Kohn, Phys. Rev. Lett. 60, 2430 (1988).

[21] A. Sanna, J. A. Flores-Livas, A. Davydov, G. Profeta, 
K. Dewhurst, S. Sharma, and E. K. U. Gross, Journal of the Physical Society of Japan 87, 041012 (2018), https://doi.org/10.7566/JPSJ.87.041012.

[22] A. Sanna, url=https://www.condmat.de/events/correl17/manuscripts/sanna.pdf (2017).

[23] L. J. Sham and M. Schlüter, Phys. Rev. Lett. 51, 1888 (1983).

[24] G. D. Gaspari and B. L. Gyorffy, Phys. Rev. Lett. 28, 801 (1972).

[25] F. S. Khan and P. B. Allen, Phys. Rev. B 29, 3341 (1984).

[26] S. Y. Savrasov and D. Y. Savrasov, Phys. Rev. B 54, 16487 (1996).

[27] S. Y. Savrasov and O. K. Andersen, Phys. Rev. Lett. 77, 4430 (1996).

[28] J. Nagamatsu, N. Nakagawa, T. Muranaka, Y. Zenitani, and J. Akimitsu, Nature (London) 410, 63 (2001).

[29] P. Canfield and G. W. Crabtree, Physics Today 56, 34 (2003).

[30] Y. Kong, O. V. Dolgov, O. Jepsen, and O. K. Andersen, Phys. Rev. B 64, 020501 (2001).

[31] J. Kortus, I. I. Mazin, K. D. Belashchenko, V. P. Antropov, and L. L. Boyer, Phys. Rev. Lett. 86, 4656 (2001).

[32] A. Y. Liu, I. I. Mazin, and J. Kortus, Phys. Rev. Lett. 87, 087005 (2001).

[33] Choi Hyoung Joon, Roundy David, Sun Hong, Cohen Marvin L., and Louie Steven G., Nature 418, 758 (2002).

[34] J. M. An and W. E. Pickett, Phys. Rev. Lett. 86, 4366 (2001).

[35] L. Boeri, J. Kortus, and O. K. Andersen, Phys. Rev. Lett. 93, 237002 (2004).

[36] K.-W. Lee and W. E. Pickett, Phys. Rev. Lett. 93, 237003 (2004).

[37] H. Rosner, A. Kitaigorodsky, and W. E. Pickett, Phys. Rev. Lett. 88, 127001 (2002).

[38] G. Savini, A. C. Ferrari, and F. Giustino, Phys. Rev. Lett. 105, 037002 (2010).

[39] E. A. Ekimov, V. A. Sidorov, E. D. Bauer, N. Mel'nik, N. J. Curro, J. Thompson, and S. Stishov, Nature 428, $542(2004)$.

[40] X. Blase, E. Bustarret, C. Chapelier, T. Klein, and C. Marcena, Nature Mater. 8, 375 (2009).

[41] Weller Thomas E., Ellerby Mark, Saxena Siddharth S., Smith Robert P., and Skipper Neal T., Nature Physics 1, 39 (2005).

[42] P. F. McMillan, Nat. Mat. 1, 1476 (2002).

[43] C. Buzea and K. Robbie, Superconductor Science and Technology 18, R1 (2005).

[44] G. Profeta, C. Franchini, N. N. Lathiotakis, A. Floris, A. Sanna, M. A. L. Marques, M. Lüders, S. Massidda, E. K. U. Gross, and A. Continenza, Phys. Rev. Lett. 96, 047003 (2006).

[45] M. Monni, F. Bernardini, A. Sanna, G. Profeta, and S. Massidda, Phys. Rev. B 95, 064516 (2017).

[46] W. S. M. and C. Richard, Nature Materials 7, 937 (2008).

[47] M. I. Eremets, V. V. Struzhkin, H.-k. Mao, and R. J. Hemley, Science 293, 272 (2001).

[48] T. Yabuuchi, T. Matsuoka, Y. Nakamoto, and K. Shimizu, Journal of the Physical Society of Japan 75, 083703 (2006), https://doi.org/10.1143/JPSJ.75.083703.

[49] Y. Ma, J. S. Tse, D. D. Klug, and R. Ahuja, Phys. Rev. B 70, 214107 (2004).

[50] Y. Yao, D. D. Klug, J. Sun, and R. Martoňák, Phys. Rev. Lett. 103, 055503 (2009).
[51] B. I. Min, H. J. F. Jansen, and A. J. Freeman, Phys. Rev. B 30, 5076 (1984).

[52] E. Maksimov and D. Savrasov, Solid State Communications 119, 569 (2001).

[53] C. J. Pickard and R. J. Needs, Nature Physics 3, 473 EP (2007).

[54] P. Cudazzo, G. Profeta, A. Sanna, A. Floris, A. Continenza, S. Massidda, and E. K. U. Gross, Phys. Rev. Lett. 100, 257001 (2008).

[55] P. Cudazzo, G. Profeta, A. Sanna, A. Floris, A. Continenza, S. Massidda, and E. K. U. Gross, Phys. Rev. B 81, 134505 (2010).

[56] P. Cudazzo, G. Profeta, A. Sanna, A. Floris, A. Continenza, S. Massidda, and E. Gross, Phys. Rev. B 81, 134506 (2010).

[57] J. M. McMahon and D. M. Ceperley, Phys. Rev. B 84, 144515 (2011).

[58] M. Borinaga, I. Errea, M. Calandra, F. Mauri, and A. Bergara, Phys. Rev. B 93, 174308 (2016).

[59] E. G. Dalladay-Simpson, R. T. Howie, Nature 529, 63 (2016).

[60] M. Eremets, I. Troyan, and A. Drozdov, arXiv/cond-mat 1601.04479 .

[61] R. P. Dias and I. F. Silvera, Science 355, 715 (2017).

[62] J. M. McMahon, M. A. Morales, C. Pierleoni, and D. M. Ceperley, Rev. Mod. Phys. 84, 1607 (2012).

[63] I. Errea and M. Calandra and C. J. Pickard and J. R. Nelson and R. J. Needs and Y. Li and H. Liu and Y. Zhang and Y. Ma and F. Mauri, Nature 532, 81 (2016).

[64] J. Yang, A. Sudik, C. Wolverton, and D. J. Siegel, Chem. Soc. Rev. 39, 656 (2010).

[65] Http://energy.gov/eere/fuelcells/hydrogen-storage.

[66] M. I. Eremets, I. A. Trojan, S. A. Medvedev, J. S. Tse, and Y. Yao, Science 319, 1506 (2008), http://science.sciencemag.org/content/319/5869/1506.full.pdf.

[67] A. van de Walle and G. Ceder, Journal of Phase Equilibria 23, 348 (2002).

[68] E. Zurek, R. Hoffmann, N. Ashcroft, A. R. Oganov, and A. O. Lyakhov, Proc. Natl. Acad. Sci. U.S.A 106, 17640 (2009).

[69] A. P. Drozdov, M. E. M. I., I. Troyan, V. Ksenofontov, and S. Shylin, Nature 525, 73 (2015).

[70] N. Bernstein, C. S. Hellberg, M. D. Johannes, I. I. Mazin, and M. J. Mehl, Phys. Rev. B 91, 060511 (2015).

[71] C. Heil and L. Boeri, Phys. Rev. B 92, 060508 (2015).

[72] D. Duan, Y. Liu, F. Tian, D. Li, X. Huang, Z. Zhao, H. Yu, B. Liu, W. Tian, and T. Cui, Sci. Rep. 4, 6968 (2014).

[73] Y. Fu, X. Du, L. Zhang, F. Peng, M. Zhang, C. J. Pickard, R. J. Needs, D. J. Singh, W. Zheng, and Y. Ma, Chem. Mater 28, 1746 (2016).

[74] M. Eremets, Private Communication.

[75] J. A. Flores-Livas, M. Amsler, C. Heil, A. Sanna, L. Boeri, G. Profeta, C. Wolverton, S. Goedecker, and E. K. U. Gross, Phys. Rev. B 93, 020508 (2016).

[76] A. Drozdov, M. I. Eremets, and I. A. Troyan, arXiv/cond-mat/1508.06224 (2015).

[77] H. Wang, J. S. Tse, K. Tanaka, T. Iitaka, and Y. Ma, Proc. Natl. Acad. Sci. U.S.A 109, 6463 (2012).

[78] T. Bi, N. Zarifi, T. Terpstra, and E. Zurek, arXiv preprint, arXiv:1806.00163 (2018).

[79] C. M. Pépin, G. Geneste, A. Dewaele, M. Mezouar, and P. Loubeyre, Science 357, 382 (2017).

[80] L. B. Christoph Heil, Giovanni B. Bachelet, arXiv 
preprint, arXiv:1804.03572 (2018).

[81] C. Heil, S. Di Cataldo, G. B. Bachelet, and L. Boeri, .

[82] C. Kokail, W. von der Linden, and L. Boeri, Phys. Rev. Materials 1, 074803 (2017).

[83] J. A. Flores-Livas, A. Sanna, A. Davydov, S. Goedecker, and M. A. L. Marques, arxiv-condmat/1610.04110 .

[84] Flores-Livas, José A., Grauzinyte, Migle, Boeri, Lilia, Profeta, Gianni, and Sanna, Antonio, Eur. Phys. J. B
91, 176 (2018).

[85] I. I. Naumov, R. J. Hemley, R. Hoffmann, and N. W. Ashcroft, The Journal of Chemical Physics 143, 064702 (2015), doi: 10.1063/1.4928076.

[86] C. Kokail, C. Heil, and L. Boeri, Phys. Rev. B 94, 060502 (2016).

[87] A. Bhaumik, R. Sachan, and J. Narayan, ACS Nano 11, 5351 (2017), pMID: 28448115, https://doi.org/10.1021/acsnano.7b01294. 